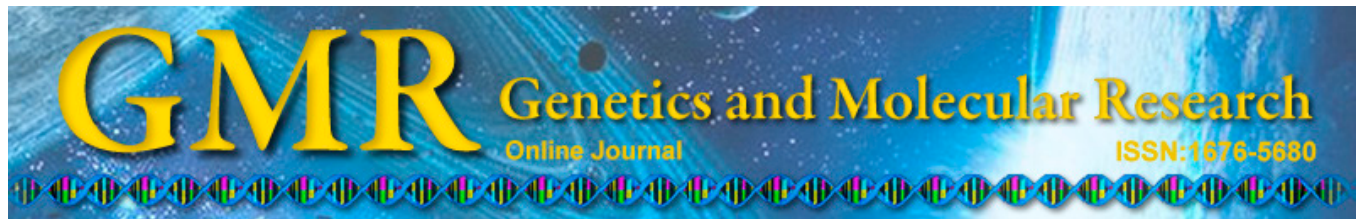

\title{
Cloning and characterization of a benzoic acid/salicylic acid carboxyl methyltransferase gene involved in floral scent production from lily (Lilium 'Yelloween')
}

\author{
H. Wang*, M. Sun*, L.L. Li, X.H. Xie and Q.X. Zhang \\ Beijing Key Laboratory of Ornamental Plants Germplasm Innovation \& \\ Molecular Breeding, National Engineering Research Center for Floriculture, \\ Beijing Laboratory of Urban and Rural Ecological Environment and College \\ of Landscape Architecture, Beijing Forestry University, Beijing, China \\ *These authors contributed equally to this study. \\ Corresponding author: Q.X. Zhang \\ E-mail: zqxbjfu@126.com
}

Genet. Mol. Res. 14 (4): 14510-14521 (2015)

Received June 30, 2015

Accepted September 26, 2015

Published November 18, 2015

DOI http://dx.doi.org/10.4238/2015.November.18.14

\begin{abstract}
In lily flowers, the volatile ester methyl benzoate is one of the major and abundant floral scent compounds; however, knowledge regarding the biosynthesis of methyl benzoate remains unknown for Lilium. In this study, we isolated a benzoic acid/salicylic acid carboxyl methyltransferase (BSMT) gene, LiBSMT, from petals of Lilium 'Yelloween'. The gene has an open reading frame of 1083 base pairs (bp) and encodes a protein of $41.05 \mathrm{kDa}$. Sequence alignment and phylogenetic analyses of LiBSMT revealed $40-50 \%$ similarity with other known benzenoid carboxyl methyltransferases in other plant species, and revealed homology to BSMT of Oryza sativa. Heterologous expression of this gene in Escherichia coli yielded an enzyme responsible for catalyzing benzoic acid and salicylic acid to methyl benzoate and methyl salicylate, respectively. Quantitative real-
\end{abstract}


time polymerase chain reaction analysis showed that $L i B S M T$ was preferentially expressed in petals. Moreover, the expression of LiBSMT in petals was developmentally regulated. These expression patterns correlate well with the emission of methyl benzoate. Our results indicate that $L i B S M T$ plays an important role in floral scent methyl benzoate production and emission in lily flowers.

Key words: Benzoic acid/salicylic acid carboxyl methyltransferase; Floral scent; Lilium 'Yelloween'; Methyl benzoate; Methyl salicylate

\section{INTRODUCTION}

Floral scent is one of the most important characteristics of ornamental plants and cut flowers (Chandler and Bugliera, 2011), plays a significant role in plant-insect interactions and attracting pollinators (Dudareva et al., 2000a), and thus in determining the seed and fruit set (Hsiao et al., 2006). Scent can also be a strategy for defense of plants, acting as signals responding to injure and harm (Gang, 2005). Moreover, floral scent has important commercial value and appeal, improving consumers' buying desire (Oyama-Okubo et al., 2011). For example, a survey of sweet pea (Lathyrus odoratus) showed that purchasers consider aroma to be more attractive than flower shape or color (Sexton et al., 2005). Floral scent is made up of various volatile organic compounds (VOCs), and more than 1700 VOCs have been identified, most of which include terpenoids, benzenoids, and fatty acid derivatives (Knudsen et al., 2006; Pichersky et al., 2006; Dudareva et al., 2013).

Methyl benzoate (MeBA) and methyl salicylate (MeSA) are common volatile esters in the plant kingdom that widely contribute to the total floral scent output and play an important role as attractants for pollinators and plant defense (Dobson, 1994; Hippauf et al., 2010). These chemicals are synthesized by benzoic acid carboxyl methyltransferase (BAMT) and salicylic acid carboxyl methyltransferase (SAMT), catalyzing the transfer of the methyl donor $S$-adenosyl-L-methionine (SAM) to corresponding acids. These 2 enzymes belong to SABATH family, a new class of $O$-methyltransferases and $N$-methyltransferases. They are named based on the first 3 identified enzymes, including SAMT, BAMT, and theobromine synthase (D'Auria et al., 2003). The first member of the SABATH family identified to be responsible for floral MeSA synthesis was from Clarkia breweri (Ross et al., 1999), followed by BAMT from snapdragon (Antirrhinum majus) (Murfitt et al., 2000). BAMT can specifically catalyze benzoate (BA) to MeBA (Dudareva et al., 2000a; Murfitt et al., 2000) and SAMT catalyzes salicylic acid (SA) to MeSA (Ross et al., 1999). Nevertheless, some SAMTs possess BAMT activity; they can catalyze the conversion of both SA and BA. This type of enzyme is referred to as BSMT.

To date, genes encoding SAMT, BAMT, and BSMT related to floral scent have been isolated and charcterized from many model plants, including C. breweri (Ross et al., 1999), A. majus (Murfitt et al., 2000), Petunia hybrida (Negre et al., 2003), Stephanotis floribunda (Pott et al., 2004), Hoya carnosa (Effmert et al., 2005), Nicotiana suaveolens, and N. alata (Pott et al., 2004; Hippauf et al., 2010). In most of these species, the gene sequences shared $38-99 \%$ similarity on the amino acid level, and the molecular mass of the proteins varied from 39.9-43.7 kDa (Effmert et al., 2005). Most of these genes are involved in producing MeBA and/or MeSA as floral scent compounds, and are highly and specifically expressed in petal tissues where scent compounds are produced and emmitted. However, no lily BAMT and 
SAMT gene has been isolated, and no genes related to floral scent of lilies have been cloned and characterized.

Lily is one of the most popular cut flowers and plays a central role in the flower market worldwide (Lee et al., 2011). After floral color and shape, fragrance is another major characteristic of ornamental lily. Previous studies have focused on the identification and emission patterns of volatiles in lily flowers. These studies showed that monoterpenoids and benzenoids were the major compounds of lily scent (Morinaga et al., 2009; Kong et al., 2012, 2013) and petals are the main emission source of fragrance compounds, while other floral parts released little or no volatiles (Kong et al., 2012). Additionally, scent emission was regulated during the flowering stages (Zhang et al., 2013). Although numerous experiments have examined lily scents, studies on their biosynthesis are lacking.

Lilium 'Yelloween', a popular OT (oriental $\mathrm{x}$ trumpet) hybrid cultivar in the market, has large yellow flower with fragrant, flowery, and musky floral scents. Our previous study showed that 'Yelloween' emitted higher levels of MeBA and lower levels of MeSA, accounting for up 30 and $6 \%$ of total floral scent components, respectively (Kong et al., 2012), but no information regarding the genes and enzymes involved in these 2 compounds has been published. In this study, we isolated a LiBSMT from petals of Lilium 'Yelloween' and successfully expressed the protein in Escherichia coli. Its function was preliminarily characterized by analyzing catalytic products of crude proteins in the medium. Tissue-specific and developmental expression of LiBSMT was further determined to analyze the correlation between gene expression and scent emission. This is the first study to clone and characterize the BSMT gene from Lilium, which may contribute to the biotechnological regulation of scent in Lilium plants.

\section{MATERIAL AND METHODS}

\section{Plant materials}

Fresh lily cut flowers of Lilium 'Yelloween' were collected from the field in Xiaotangshan, Beijing. Flowers were placed in deionized water and kept in a controlled-environment chamber at $24^{\circ} \pm 1{ }^{\circ} \mathrm{C}$ with a 12 -h light/12-h dark cycle. Light intensity was maintained at 150 $\pm 5 \mu \mathrm{mol} \cdot \mathrm{m}^{-2} \cdot \mathrm{s}^{-1}$ and a relative air humidity of $60 \%$. All reagents were from Sigma-Aldrich (St. Louis, MO, USA) unless otherwise stated.

\section{RNA extraction and isolation of LiBSMT}

Total RNA was isolated from frozen petals using the EASYspin plus plant RNA kit (Aidlab, Beijing, China) following the manufacturer protocols. To clone the gene fragments, a SMARTer ${ }^{\mathrm{TM}}$ rapid amplification of cDNAs ends (RACE) cDNA amplification kit (Clontech, Mountain View, CA, USA) and the Advantage 2 cDNA Polymerase Mix (Clontech) were used to isolate the 3' and 5' ends of the cDNA. Gene-specific primers (GSP1 for 5'-RACE and GSP2 for 3'-RACE) were designed based on the sequence obtained from a transcriptome of lily flowers. A universal primer was used for both 5'- and 3'-RACE (Table 1). RACE amplifications used a touchdown polymerase chain reaction (PCR) program: 5 cycles of $94^{\circ} \mathrm{C}$ for $30 \mathrm{~s}, 72^{\circ} \mathrm{C}$ for $2 \mathrm{~min} ; 5$ cycles of $94^{\circ} \mathrm{C}$ for $30 \mathrm{~s}, 70^{\circ} \mathrm{C}$ for $30 \mathrm{~s}, 72^{\circ} \mathrm{C}$ for $2 \mathrm{~min} ; 27$ cycles of $94^{\circ} \mathrm{C}$ for $30 \mathrm{~s}, 68^{\circ} \mathrm{C}$ for $30 \mathrm{~s}$, and $72^{\circ} \mathrm{C}$ for $2 \mathrm{~min}$. The amplified cDNA fragments were purified using a gel extraction kit (Biomega, San Diego, CA, USA) and subcloned into the pMD18-T 
vector (Takara, Dalian, China), transformed into E. coli DH5a (Tiangen, Beijing, China), and selected by colony PCR. The plasmids were extracted and sequenced.

\begin{tabular}{ll} 
Table 1. Primer sequences used in this study. \\
\hline Primer codes & Sequence $\left(5^{\prime} \rightarrow 3^{\prime}\right)$ \\
\hline 5'- and $3^{\prime}-$ RACE & \\
GSP1 & AGAGGGAGGGAAGAGAGACCGTGCCATT \\
GSP2 & GTATCCAGATGGCTTTCTCAGGTTCCTCG \\
Universal primer & CTAATACGACTCACTATAGGGCAAGCAGTGGTATCAACGCAGAGT \\
Isolation of full-length cDNA & TACATGGTTGGCGGGAATG \\
LiF-1 & ATACAACCACAATTAAGAACAAC \\
LiR-1 & \\
Construction of expression vector & CATATGGTTGGCGGGAATGGTGAAAC \\
LiF-2 & GGATCCTCATGCTTTCTTCTCTAAGG \\
LiR-2 & \\
Real-time PCR & CCTCCCAGGAAACGACTTCA \\
LiF-3 & GAACCAGGAACCCCACTAC \\
LiR-3 & TGTGCTTTCCTCTACGCCAGT \\
LhActin-F & TCCCTCACGATTCCCGCTCT \\
LhActin- - &
\end{tabular}

Sequences underlined indicate restriction sites.

The full-length cDNA sequence was obtained by splicing of the 5'-RACE fragment, partial cDNA fragment, and 3'-RACE fragment. A pair of primers designed according to the end of $5^{\prime}$ and 3 ' sequences were used to amplify the full-length gene (Table 1). The amplified fragment was sequenced to determine that the correct sequence had been obtained. The PCR program was run under following conditions: initial denaturation at $94^{\circ} \mathrm{C}$ for $5 \mathrm{~min}$, followed by 30 cycles of denaturation at $94^{\circ} \mathrm{C}$ for $30 \mathrm{~s}$, annealing at $58^{\circ} \mathrm{C}$ for $30 \mathrm{~s}$, extension at $72^{\circ} \mathrm{C}$ for $2 \mathrm{~min}$, and a final extension for $10 \mathrm{~min}$.

\section{DNA sequencing and analysis}

Primers were designed using the Primer Premier 5 software. DNA fragments were sequenced at Beijing Ruibiotech Biotechnology Co., Ltd. (Beijing, China). The coding sequences were identified by using the NCBI Database. Multiple sequence alignment was performed using the DNAMAN software. Prediction of the LiBSMT open reading frame (ORF) was carried out using ORF Finder (http://www.ncbi.nlm.nih.gov/projects/gorf/). A phylogenetic tree was constructed using the MEGA5 software based on ClustalX alignment, and bootstrap values were inferred from 1000 replicates.

\section{Construction of $L i B S M T$ expression vector and prokaryotic expression}

For heterologous expression of LiBSMT, a recombinant expression vector was constructed. The protein-coding region of the gene was amplified using the forward and reverse primer containing the NdeI and BamHI restriction sites (Table 1). The amplified product was cloned into the pMD19-T vector. After double-digestion with NdeI and BamHI (New England Biolabs, Ipswich, MA, USA), the fragment was cloned into the NdeI-BamHI site of the expression vector pET-28a (+) (Novagen, Madison, WI, USA) with an N-terminal (His) ${ }_{6}$-tag. Sequence analysis indicated that no errors had been introduced. Then the constructed plasmid 
and plasmid without the insert were transformed into E. coli BL21 (DE3) cells (Tiangen), and cultured in Luria-Bertani (medium containing $50 \mu \mathrm{g} / \mathrm{mL}$ kanamycin at $37^{\circ} \mathrm{C}$ with vigorous shaking. When $\mathrm{A}_{600}$ of the culture density reached approximately $0.6-0.8$, isopropyl $\beta$-D-thiogalactopyranoside (IPTG) was added at a final concentration of $1 \mathrm{mM}$ to induce the expression of BSMT. After $6 \mathrm{~h}$ incubation with shaking at $200 \mathrm{rpm}$ at $37^{\circ} \mathrm{C}, \mathrm{E}$. coli cells were collected by centrifugation and boiled in $5 \mathrm{X}$ sodium dodecyl sulfate-polyacrylamide gel electrophoresis (SDS-PAGE) loading buffer (10\% SDS, $1 \mathrm{M}$ Tris-HCl, $\mathrm{pH} 6.8,0.1 \%$ bromophenol blue, $50 \%$ glycerol, and $60 \mathrm{mM} \beta$-mercaptoethanol) for $5 \mathrm{~min}$. Overexpressed proteins were separated by $12 \%$ SDS-PAGE.

\section{Gas chromatography-mass spectrometry (GC-MS) analysis of enzyme products}

For functional characterization, crude proteins in the medium were assayed with substrates as previously described by Hippauf et al. (2010) with some modifications. A 50-mL cell culture of $E$. coli BL21 (DE3) expressing BSMT was supplemented with $1 \mathrm{mM}$ IPTG when the $\mathrm{A}_{600}$ of the culture reached $0.6-0.8$. Next, the culture was incubated at $37^{\circ} \mathrm{C}$ for $3 \mathrm{~h}$, and then $2 \mathrm{mM}$ BA only, SA only, or a combination of $2 \mathrm{mM} \mathrm{BA}$ and $2 \mathrm{mM}$ SA were added. To assess the preference for either SA or BA of the enzyme, reactions were performed under saturating substrate concentrations (Barkman et al., 2007). Our initial studies suggested several concentrations $(1,2,4$, and $8 \mathrm{mM})$ of substrate, and $2 \mathrm{mM}$ substrate showed the highest levels of products formation over time (data not shown). After incubation with substrates for $1 \mathrm{~h}$, cells were removed by centrifugation, and the remaining supernatant was extracted with $3 \mathrm{~mL}$ hexane. Samples were analyzed by GC-MS on a GC-MS-QP2010 Ultra (Shimadzu, Kyoto, Japan). GC was performed on a capillary column (Rtx-5MS, $30 \mathrm{~m}$ x $0.25 \mathrm{~mm}$ x 0.25 $\mu \mathrm{m}$; Shimadzu) at a flow rate of $1 \mathrm{~mL} / \mathrm{min}$ under helium gas. The injector port temperature was set at $200^{\circ} \mathrm{C}$. The $\mathrm{GC}$ oven temperature was $35^{\circ} \mathrm{C}$ for $2 \mathrm{~min}$, and then the temperature was increased at a rate of $10^{\circ} \mathrm{C} / \mathrm{min}$ to $280^{\circ} \mathrm{C}$, and finally held for $10 \mathrm{~min}$.

The GCMS Postrun Analysis software was used for volatile compounds analysis. Products were identified by comparison of mass spectra to those in the library of National Institute of Standards and Technology 11 and retention index. Benzyl alcohol was used as an external standard to quantify the products of the enzyme reaction. Benzyl alcohol was dissolved in hexane in a series of concentrations and analyzed using the method described above.

\section{Quantitative real-time PCR analysis}

To perform quantitative real-time PCR (qRT-PCR) analysis, total RNA was isolated from different floral parts and petals during the lifespan of the flower using the EASYspin plus plant RNA kit (Aidlab). A total of $1 \mu \mathrm{g}$ RNA was used to prepare cDNA using a Transcriptor first-strand cDNA synthesis kit (Tiangen). Primers for qRT-PCR were designed using an on-line primer designing tool (http://www.ncbi.nlm.nih.gov/tools/primer-blast/) based on the LiBSMT cDNA sequence. LhActin (GenBank accession No. AB438963) was selected as internal reference gene for each sample (Table 1). qRT-PCR was carried out using the BioRad real-time PCR system (Bio-Rad, Hercules, CA, USA) with SYBR ${ }^{\circledR}$ Premix Ex Taq ${ }^{\mathrm{TM}}$ kit (Takara). A volume of $10 \mu \mathrm{L}$ containing $0.2 \mu \mathrm{L} 10 \mu \mathrm{M}$ forward primer, $0.2 \mu \mathrm{L} 10 \mu \mathrm{M}$ reverse primer, $5 \mu \mathrm{L} 2 \mathrm{X}$ SYBR green premix, $1 \mu \mathrm{L}$ cDNA template, and $3.6 \mu \mathrm{L} \mathrm{ddH_{2 }} \mathrm{O}$ was used for PCR amplification. The program was performed as follows: $95^{\circ} \mathrm{C}$ for $30 \mathrm{~s} ; 40$ cycles of $95^{\circ} \mathrm{C}$ 
for $5 \mathrm{~s}, 58^{\circ} \mathrm{C}$ for $30 \mathrm{~s}$, and $72^{\circ} \mathrm{C}$ for $30 \mathrm{~s}$; followed by melting curve analysis at $60^{\circ} \mathrm{C}$ for 30 $\mathrm{s}$ then ramping at $7^{\circ} \mathrm{C} / \mathrm{s}$ to $95^{\circ} \mathrm{C}$. Relative levels of gene expression were analyzed using the CFX Manager 3.0 Software and normalized with the results for the LhActin gene.

\section{RESULTS}

\section{Isolation and sequence analysis of LiBSMT}

Using RACE technology, we obtained a full-length cDNA encoding BSMT from the petals of Lilium 'Yelloween'. The full-length putative gene was named LiBSMT and registered in GenBank (Accession No. KJ755672). LiBSMT contained an ORF of 1083 bp with a 4-bp 5'-untranslated region (UTR) and 83-bp 3'-UTR. This gene was predicted to encode a protein of 360 amino acids with a calculated molecular mass of $41.05 \mathrm{kDa}$ and theoretical isoelectric point of 5.67 using the online tool ProtParam (http://web.expasy.org/protparam/).

A BLAST search of NCBI revealed that the amino acid sequence of LiBSMT contained a methyltransf_7 domain, which is the characteristic domain of methyltransferases. Alignment of the deduced amino acid sequence encoded by LiBSMT with carboxyl methyltransferases from several other species showed that LiBSMT shared 40-50\% similarity with sequences from A. majus, C. breweri, N. suaveolens, O. sativa, and P. hybrida (Figure 1).

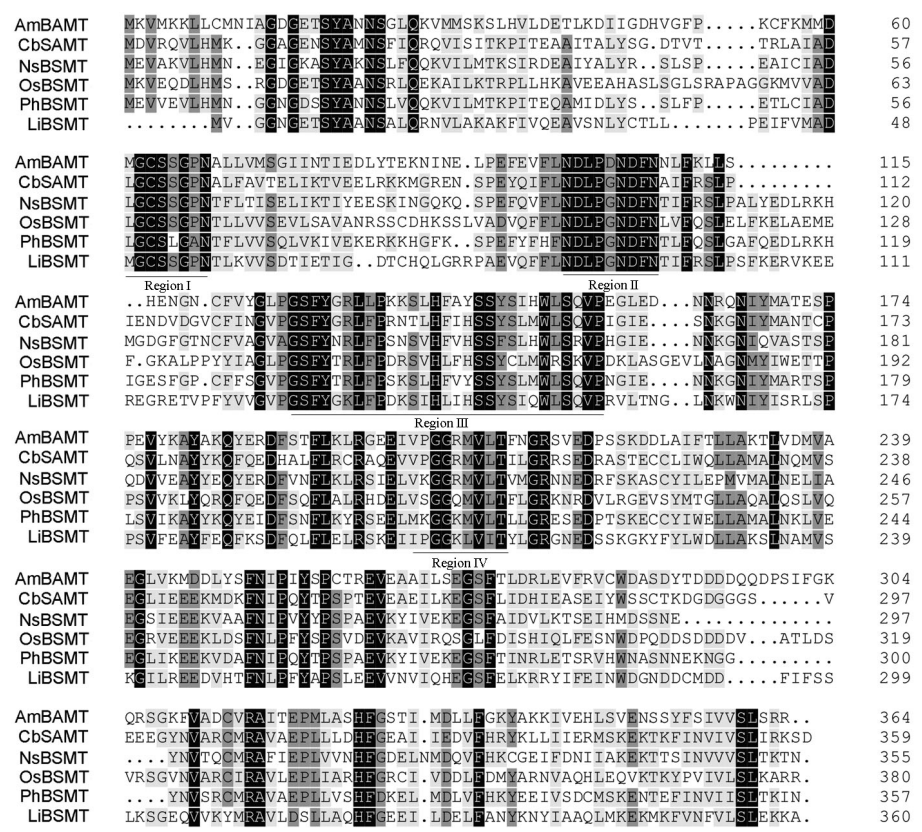

Figure 1. Alignment of predicted amino acid sequence of Lilium 'Yelloween' BSMT with other closely related plant carboxyl methyltransferases. Sequence alignment was performed using the DNAMAN software. The numbers on the right side indicate the amino acid positions in the sequence. Amino acids in black backgrounds indicate identical residues. Dashes indicate gaps that have been inserted for optimal alignment. The 4 highly identical sequences are underlined. GenBank accession numbers are as follows: AmBAMT (Antirrhinum majus BAMT, AAF98284.1), CbSAMT (Clarkia breweri SAMT, AAF00108.1), NsBSMT (Nicotiana suaveolens BSMT, CAF31508.1), OsBSMT (Oryza sativa, XM467504, MAFF GenBank), PhBSMT1 (Petunia hybrida BSMT1, AAO45012.1), and LiBSMT (Lilium 'Yelloween' BSMT KJ755672). 
A phylogenetic tree was constructed based on the LiBSMT and other enzymatically characterized SABATH gene family members related to emission of benzenoid esters, and several clades were identified. The newly-identified LiBSMT appeared to be related to OsBSMT, and they were clustered into the same subgroup (Figure 2).

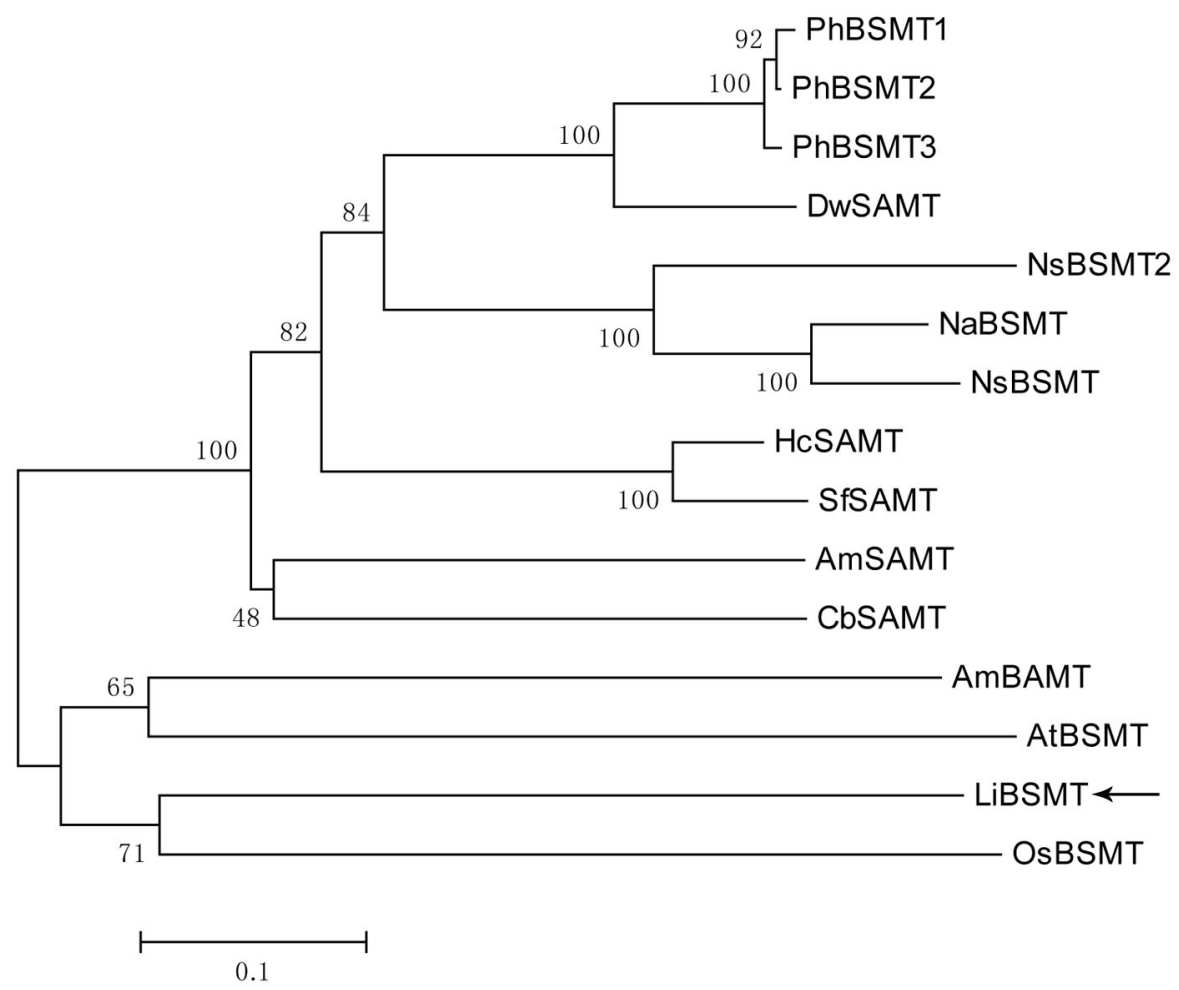

Figure 2. Phylogenetic analysis of the deduced amino acid sequence of LiBSMT and other SABATH gene family members. Phylogenetic tree for LiBSMT protein was constructed using the neighbor-joining method with the MEGA 5 software. Percentages of bootstrap values of 1000 replicates are presented at each branch point. Abbreviation of each protein and GenBank accession numbers are as follows; PhBSMT1 from Petunia hybrida (AAO45012.1), PhBSMT2 from P. hybrida (AAO45013.1), PhBSMT3 from $P$. hybrida (ABF50941.1), DwSAMT from Datura wrightii (ABO71015.1), NsBSMT2 from Nicotiana suaveolens (ACZ55217.1), NaBSMT from $N$. alata (ACZ5520.1), NsBSMT from N. suaveolens (CAF31508.1), HcSAMT from Hoya carnosa (CAI05934.1), SfSAMT from Stephanotis floribunda (CAC33768.1), AmSAMT from Antirrhinum majus (AAN40745.1), CbSAMT from Clarkia breweri (AAF00108.1), AmBAMT from A. majus (AAF98284.1), AtBSMT from Arabidopsis thaliana (AAY25461.1), LiBSMT from Lilium 'Yelloween' (KJ755672), and OsBSMT from Oryza sativa (XM467504, MAFF GenBank).

\section{Overexpression of LiBSMT in $E$. coli}

To determine whether the gene isolated from lily flower encoded an enzyme with BSMT activity, the coding region of LiBSMT gene was subcloned into an expression vector and expressed in E. coli. SDS-PAGE analysis showed a strengthened protein band at the 40$\mathrm{kDa}$ protein marker, which was identical to the predicted molecular mass from the sequence of LiBSMT (Figure 3). 


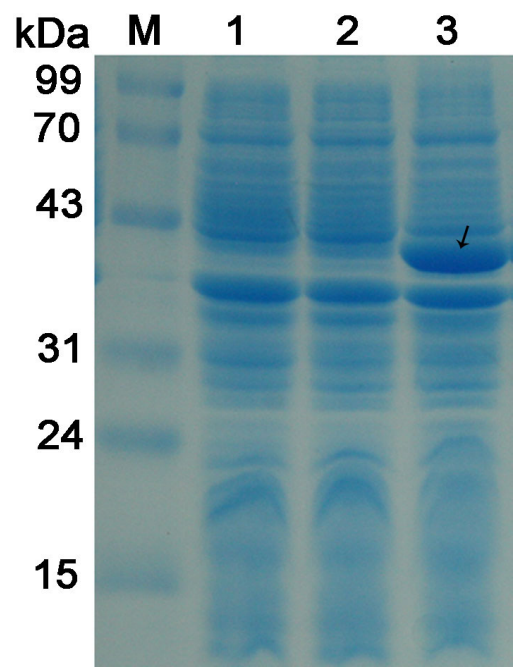

Figure 3. SDS-PAGE (12\%) analysis of fusion protein expression in Escherichia coli. Lane $M=$ protein marker; lane 1 = supernatant of pET-28a without IPTG induction; lane 2 = supernatant of pET-28a-LiBSMT cells without IPTG induction; lane 3 = supernatant of pET-28a-LiBSMT cells with IPTG induction.

\section{Characterization of LiBSMT expressed in E. coli}

For functional characterization of the identified $B S M T$ gene from lily, the growth medium of $E$. coli cultures was supplied with substrates then extracted with hexane, and the extracts were analyzed by GC-MS. Figure 4 shows the reaction products of the enzyme. After BA and/or SA was added to cell lysates, MeBA and/or MeSA were detected. Single feeding experiments showed that BSMT produced similar levels of MeBA and MeSA when the growth medium was supplemented with only BA or SA (Figure 4A, B, F). However, when the medium was supplemented with equivalent amounts of BA and SA at the same time, MeBA production was more than 3 -fold higher than MeSA production (Figure 4C, F). In contrast, the medium of induced culture without substrates and E. coli cells carrying a pET-28a plasmid with no insert contained neither of these 2 esters (Figure 4D, E, F).

\section{Tissue-specific and developmental expression pattern of $\mathrm{LiBSMT}$}

The qRT-PCR results showed that transcripts differed in floral parts (petals, anthers, filaments, stigma) and leaves of 'Yelloween'. The highest level of LiBSMT expression was observed in the petals, and a relatively lower transcript level was detected in the anthers. A very low transcript level was detected in the stigma and filaments. No LiBSMT expression was found in the leaves (Figure 5A). Because transcripts were mostly found in petals, we examined the expression pattern during flower developmental stages. During the lifespan of the flower, the expression signal was first detected in the mature bud (1 day before opening), and its level increased rapidly during the first 3 days and peaked on day 4 after anthesis, when the flower was fully opened. Over the subsequent 3 days, transcript levels declined by approximately $80 \%$ and no detectable signals were observed on days 8 and 9 (Figure 5B). 

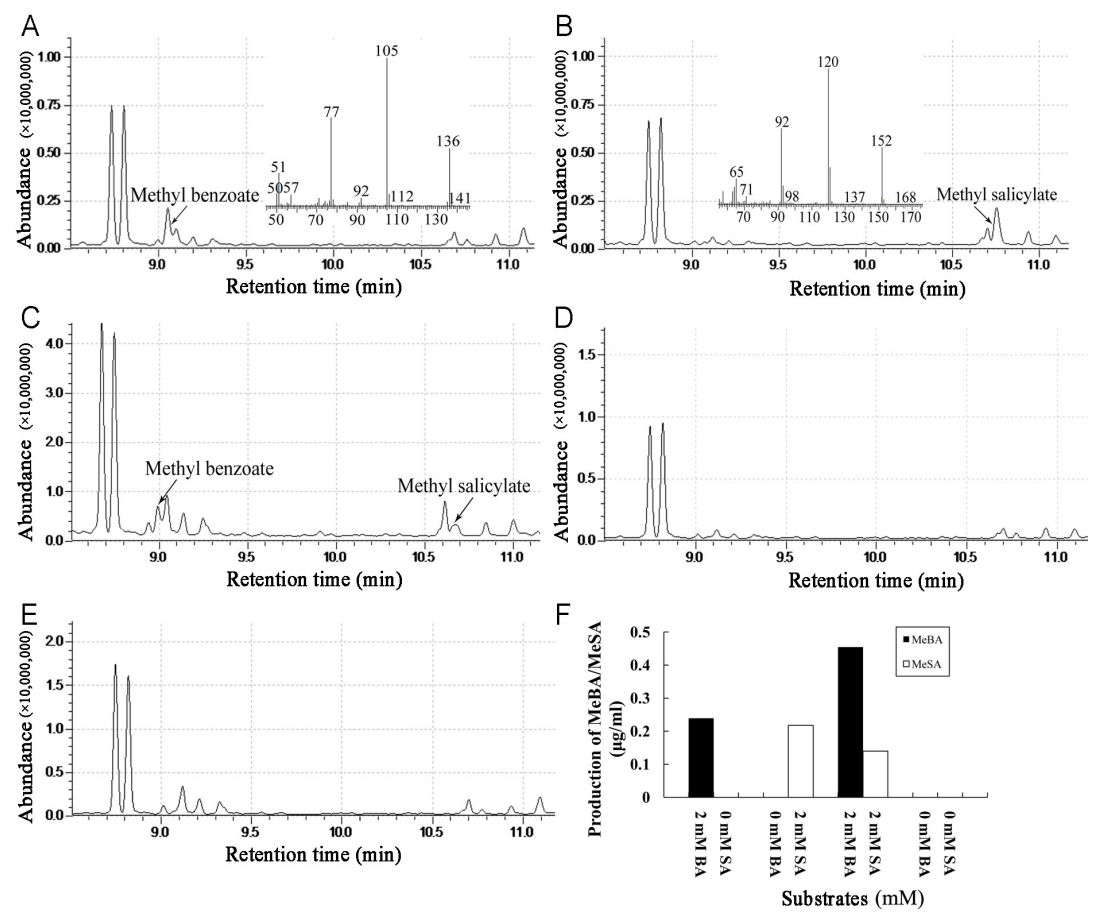

Figure 4. GC-MS analysis of reaction products formed in the medium of Escherichia coli cells expressing Lilium 'Yelloween' BSMT. A. Analysis of the medium in which E. coli cells expressed BSMT after induction with IPTG. The medium was supplemented with only $2 \mathrm{mM}$ BA. B. Analysis of the medium in which $E$. coli cells expressed BSMT after induction with IPTG. The medium was supplemented with only $2 \mathrm{mM} \mathrm{SA}$. C. Analysis of the medium in which $E$. coli cells expressed BSMT after induction with IPTG. The medium was supplemented with 2 mM BA and 2 $\mathrm{mM}$ SA. D. Analysis of the medium in which $E$. coli cells expressed BSMT after induction with IPTG. The medium was not supplemented with BA or SA. E. Analysis of the medium in which E. coli cells expressed pET-28a vector with no insert after induction with IPTG. The medium was not supplemented with BA or SA. F. Amounts of MeBA/ MeSA formed in the medium in which E. coli cells expressed BSMT. All experiments were replicated 3 times, and representative figures are shown. Insets in A and B show the mass spectra for MeBA and MeSA, respectively.

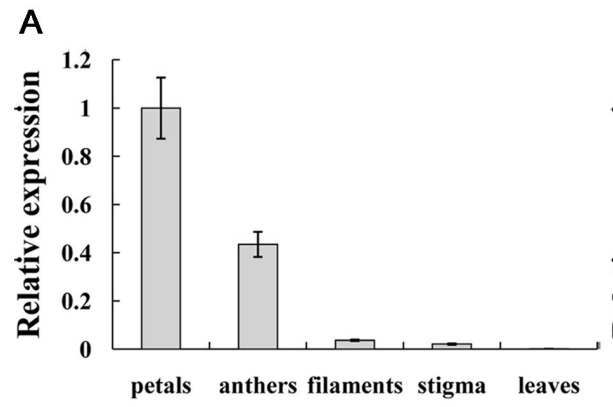

Tissue type
B

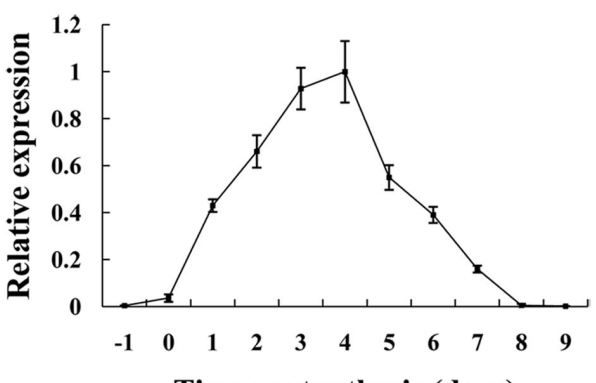

Time postanthesis (days)

Figure 5. Expression analyses of LiBSMT in Lilium 'Yelloween' by real-time PCR. A. Expression analysis of LiBSMT in different floral parts of 4-day old lily flowers and leaves. B. Expression levels of LiBSMT in petals during the lifespan of flower, ' -1 ' represents 2 days before flower opening and ' 0 ' represents 1 day before flower opening. Samples were harvested at approximately 7 p.m. Data are reported as the means \pm SE of 3 replicates. 


\section{DISCUSSION}

Benzenoid esters are common volatile constituents in many plant species, of which methyl benzoate is one of the most abundant scent components in Lilium (Kong et al., 2012). Benzenoid carboxyl methyltransferase genes have been isolated and characterized in many plants; however, this gene in Lilium has not been isolated. In this study, the full-length cDNA of LiBSMT was cloned from the petals of Lilium 'Yelloween'. The deduced LiBSMT protein contained 4 highly conserved regions as described by Pott et al. (2002)(Figure 1). Phylogenetic analysis revealed that LiBSMT was closely related to monocot OsBSMT. The dendrogram obtained also supported that enzymes with SA/BA carboxyl methyltransferase activity may have been generated from 4 genes of independent origin, including Arabidopsis BSMT, Antirrhinum BAMT, rice BSMT, SAMT, and Solanaceae BSMT (Hippauf et al., 2010) (Figure 2).

To study the function of LiBSMT, crude proteins in the medium were assayed in the presence of substrates. Our preliminary experiments indicated that LiBSMT possessed MeBA- and MeSA-forming activity. Therefore, we predicted that LiBSMT was responsible for the synthesis of both MeBA and MeSA in flowers of 'Yelloween'. This is similar to BSMTs from other plant species (Pott et al., 2004; Barkman et al., 2007; Hippauf et al., 2010; Zhao et al., 2010). Moreover, the relatively high preference of crude enzyme for BA may result in the higher emission of MeBA (Kong et al., 2012). Further studies are needed to purify the enzyme and determine its activity for both substrates to confirm the affinity and efficiency of LiBSMT.

Previous studies demonstrated that petals are mainly responsible for volatile production and emission in the flowers of many plant species (Dobson, 1994; Dudareva et al., 2000b). In our study, LiBSMT was predominantly expressed in the petals of flowers, from which volatile compounds are typically emitted (Kong et al., 2012). A similar expression profile of $A m B A M T$ was observed in snapdragon (Dudareva et al., 2000a). However, unlike $A m B A M T$, relatively lower transcript levels of $L i B S M T$ were detected in the anthers of lily, suggesting that the protein may function to attract pollinators. Moreover, transcripts of $L i B$ $S M T$ in petals were developmentally regulated during flower opening, generally increasing and then decreasing over the lifespan (Figure 5B). Interestingly, scent emission was also regulated over the lifespan in many plants (Dudareva and Pichersky, 2000). Additionally, the amounts of MeBA emission in lily flowers followed this pattern (Zhang et al., 2013). Thus, there may be positive correlations between gene expression and MeBA emission, but this hypothesis requires further analysis.

Although more than one biochemical mechanism is responsible for the volatiles emitted from plants, the production and emission of volatiles may be regulated largely at the transcript level (Dudareva et al., 2004). Our data revealed a clear positive correlation between transcript levels and MeBA emission, indicating that gene expression of LiBSMT is related to the emission of MeBA. Based on the enzyme reaction products observed, LiBSMT is involved in the biosynthesis and emission of MeBA.

In conclusion, we cloned the carboxyl methyltransferase gene LiBSMT involved in floral scent from petals of Lilium 'Yelloween'. Its expression profiles and functional characterization agreed with the role for this gene in the production and emission of floral scent. Our results indicated that $L i B S M T$ is a putative BSMT homolog in Lilium plants that plays an important role in regulating floral scents. Understanding the biosynthesis of benzenoid esters is important for providing information regarding scent-related breeding in Lilium. 


\section{ACKNOWLEDGMENTS}

Research supported by the Beijing Higher Education Young Elite Teacher Project (\#YETP0747), the National Key Technology R\&D Program for the 12th Five-Year Plan (\#2012BAD01B07,\#2013BAD01B07), and Special Fund for Beijing Common Construction Project.

\section{REFERENCES}

Barkman TJ, Martins TR, Sutton E and Stout JT (2007). Positive selection for single amino acid change promotes substrate discrimination of a plant volatile-producing enzyme. Mol. Biol. Evol. 24: 1320-1329.

Chandler SF and Bugliera F (2011). Genetic modification in floriculture. Biotechnol. Lett. 33: 207-214.

D'Auria JC, Chen F and Pichersky E (2003). The SABATH Family of MTs in Arabidopsis thaliana and other Plant Species. In: Recent Advances in Phytochemistry (Romeo JT, ed.). Elsevier Science, New York, 253-283.

Dobson HEM (1994). Floral Volatiles in Insect Biology. In: Insect-Plant Interactions (Bernays EA, ed.). CRC Press, London, 47-81.

Dudareva N and Pichersky E (2000). Biochemical and molecular genetic aspects of floral scents. Plant Physiol. 122: 627-633.

Dudareva N, Murfitt LM, Mann CJ, Gorenstein N, et al. (2000a). Developmental regulation of methyl benzoate biosynthesis and emission in snapdragon flowers. Plant Cell 12: 949-961.

Dudareva N, Piechulla B and Pichersky E (2000b). Biogenesis of floral scent. Hort Rev. 24: 31-54.

Dudareva N, Pichersky E and Gershenzon J (2004). Biochemistry of plant volatiles. Plant Physiol. 135: 1893-1902.

Dudareva N, Klempien A, Muhlemann JK and Kaplan I (2013). Biosynthesis, function and metabolic engineering of plant volatile organic compounds. New Phytol. 198: 16-32.

Effmert U, Saschenbrecker S, Ross J, Negre F, et al. (2005). Floral benzenoid carboxyl methyltransferases: from in vitro to in planta function. Phytochemistry 66: 1211-1230.

Gang DR (2005). Evolution of flavors and scents. Annu. Rev. Plant Biol. 56: 301-325.

Hippauf F, Michalsky E, Huang R, Preissner R, et al. (2010). Enzymatic, expression and structural divergences among carboxyl $O$-methyltransferases after gene duplication and speciation in Nicotiana. Plant Mol. Biol. 72: 311-330.

Hsiao YY, Tsai WC, Kuoh CS, Huang TH, et al. (2006). Comparison of transcripts in Phalaenopsis bellina and Phalaenopsis equestris (Orchidaceae) flowers to deduce monoterpene biosynthesis pathway. BMC Plant Biol. 6: $1-14$.

Knudsen JT, Eriksson R, Gershenzon J and Stahl B (2006). Diversity and distribution of floral scent. Bot. Rev. 72: 1-120.

Kong Y, Sun M, Pan HT and Zhang QX (2012). Composition and emission rhythm of floral scent volatiles from eight lily cut flowers. J. Am. Soc. Hort. Sci. 137: 376-382.

Kong Y, Sun M, Pan HT, Zhang QX, et al. (2013). Floral scent composition of Lilium sulphureum. Chem. Nat. Comp. 49: 362-364.

Lee SI, Park KC, Song YS, Son JH, et al. (2011). Development of expressed sequence tag derived-simple sequence repeats in the genus Lilium. Genes Genom. 33: 723-733.

Morinaga SI, Kumano Y, Ota A, Yamaoka R, et al. (2009). Day-night fluctuations in floral scent and their effects on reproductive success in Lilium auratum. Popul. Ecol. 51: 187-195.

Murfitt LM, Kolosova N, Mann CJ and Dudareva N (2000). Purification and characterization of S-adenosyl-Lmethionine:benzoic acid carboxyl methyltransferase, the enzyme responsible for biosynthesis of the volatile ester methyl benzoate in flowers of Antirrhinum majus. Arch. Biochem. Biophys. 382: 145-151.

Negre F, Kish CM, Boatright J, Underwood B, et al. (2003). Regulation of methylbenzoate emission after pollination in snapdragon and petunia flowers. Plant Cell 15: 2992-3006.

Oyama-Okubo N, Nakayama M and Ichimura K (2011). Control of floral scent emission by inhibitors of phenylalanine ammonia-lyase in cut flower of Lilium cv. 'Casa Blanca'. J. Jpn. Soc. Hort. Sci. 80: 190-199.

Pichersky E, Noel JP and Dudareva N (2006). Biosynthesis of plant volatiles: nature's diversity and ingenuity. Science 311: 808-811.

Pott MB, Pichersky E and Piechulla B (2002). Evening specific oscillations of scent emission, SAMT enzyme activity, and SAMT mRNA in flowers of Stephanotis floribunda. J. Plant Physiol. 159: 925-934.

Pott MB, Hippauf F, Saschenbrecker S, Chen F, et al. (2004). Biochemical and structural characterization of benzenoid carboxyl methyltransferases involved in floral scent production in Stephanotis floribunda and Nicotiana suaveolens. Plant Physiol. 135: 1946-1955. 
Ross JR, Nam KH, D’Auria JC and Pichersky E (1999). S-adenosyl-L-methionine: salicylic acid carboxyl methyltransferase, an enzyme involved in floral scent production and plant defense, represents a new class of plant methyltransferases. Arch. Biochem. Biophys. 367: 9-16.

Sexton R, Stopford AP, Moodie WT and Porter AEA (2005). Aroma production from cut sweet pea flowers (Lathyrus odoratus): the role of ethylene. Physiol. Plantarum 124: 381-389.

Zhang HX, Leng PS, Hu ZH, Zhao J, et al. (2013). The floral scent emitted from Lilium 'Siberia' at different flowering stages and diurnal variation. Acta Hort. Sin. 40: 693-702.

Zhao N, Guan J, Ferrer JL, Engle N, et al. (2010). Biosynthesis and emission of insect-induced methyl salicylate and methyl benzoate from rice. Plant Physiol. Biochem. 48: 279-287. 